\title{
Link-state QoS routing protocol under various mobility models
}

\author{
Safaa Laqtib ${ }^{1}$, Khalid El Yassini ${ }^{2}$, Moulay Lahcen Hasnaoui ${ }^{3}$ \\ ${ }^{1,2}$ Informatics and Applications Laboratory (IA), Department of Mathematics and Computer Science, Faculty of Sciences, \\ Moulay Ismail University, Morocco \\ ${ }^{3}$ Research Team: ISIC ESTM, L2MI Laboratory, ENSAM Moulay-Ismail University, Morocco
}

\begin{tabular}{|c|c|}
\hline Article Info & ABSTRACT \\
\hline Article history: & \multirow{9}{*}{$\begin{array}{l}\text { Mobile Ad Hoc Network (MANET) consists of a group of mobile or wireless } \\
\text { nodes that are placed randomly and dynamically that causes the continual } \\
\text { change between nodes. A mobility model attempts to mimic the movement } \\
\text { of real mobile nodes that change the speed and direction with time. The } \\
\text { mobility model that accurately represents the characteristics of the mobile } \\
\text { nodes in an ad hoc network is the key to examine whether a given protocol. } \\
\text { The aim of this paper is to compare the performance of four different } \\
\text { mobility models (i.e. Random Waypoint, Random Direction, Random walk, } \\
\text { and Steady-State Random Waypoint) in MANET. These models were } \\
\text { configured with Optimized Link State Routing (OLSR) protocol under three } \\
\text { QoS (Quality of Service) metrics such as the Packet Delivery Ratio (PDR), } \\
\text { Throughput, End-to-End delay. The simulation results show the effectiveness } \\
\text { of Steady-State Random Waypoint Mobility Models and encourage further } \\
\text { investigations to extend it in order to guarantee other QoS requirements. }\end{array}$} \\
\hline Received Jan 1, 2019 & \\
\hline Revised Mar 10, 2019 & \\
\hline Accepted Apr 25, 2019 & \\
\hline Keywords: & \\
\hline MANET & \\
\hline Mobility models & \\
\hline OLSR protocol & \\
\hline Routing protocol & \\
\hline
\end{tabular}

Copyright (C) 2019 Institute of Advanced Engineering and Science. All rights reserved.

Corresponding Author:

Safaa Laqtib,

Informatics and Applications,

Laboratory (IA), Department of Mathematics and Computer Science,

Faculty of Sciences, Moulay Ismail University, Meknes, Morocco.

Email: laq.safaa@gmail.com

\section{INTRODUCTION}

MANET is a group of mobile nodes that wirelessly communicate together. This means that mobile nodes are able to communicate and share information without the need of any central device [1]. The mobile nodes can play the roles of both hosts and routers.

The lack of fixed infrastructure in a MANET causes diverse types of challenges. The biggest challenge among them is routing. Routing is the procedure used to set up an ideal and effective route between nodes. An ad hoc routing protocol is a standard, that controls how nodes decide which way to route packets between them in a mobile ad-hoc network.

The node mobility of MANET makes the network topology to change with time, and MANET performances need to be dynamically adapted to such changes. Hence, the networking and application protocol performances of mobile ad hoc networks are highly affected by the frequency of network topology changes. for this reason, the performance of MANET needs to be studied in existence of mobility models.

This paper presents an analysis study of mobility models of MANET utilizing OLSR through a case study. QoS (Quality of Service) metrics such as the Packet Delivery Ratio (PDR), Throughput, End-to-End delay are utilized to analyze the impact of mobility models on the OLSR routing protocol under two scenarios (varying the number, varying the speed of nodes). Results show that, indeed, the mobility model has a significant influence on the performance of the network.

To our knowledge, some published simulation results ignore the "steady-state" distribution, known as the stationary distribution in the probability literature, this paper focused on this distribution by using the 
steady-state random waypoint for the first time under MANET and compared it with various mobility models, namely: Random Waypoint, Random Walk and Random Direction.

The steady-state random waypoint can be considered as a recent advance in mobility modeling for mobile ad hoc networks and should take this distribution into account in recent research in this field. The remains of the paper are sorted out as follows: in section two we talk about the different mobility models utilized as a part of the simulation. At that point, we introduce the description of OLSR routing protocol in section three. Section four exhibits the diverse metrics used to the simulation and analysis. At long last, we close in section five.

\section{MOBILITY MODELS}

Several mobility models have been proposed to evaluate the performance of a mobile ad hoc network protocol.

\subsection{Random Waypoint Mobility Model}

The Random Waypoint (RWP) mobility model has been extensively used in mobile ad hoc network simulations. In this model, the mobile nodes are initially distributed randomly around the simulation area. each node is randomly choosing its location ( $\mathrm{x} 0$, y0), destination coordinates ( $\mathrm{x} 1, \mathrm{y} 1)$, speed of movement $\mathrm{S}$, and the amount of time that it will pause when it reaches the destination. the points $(\mathrm{x} 0, \mathrm{y} 0)$ and $(\mathrm{x} 1, \mathrm{y} 1)$ are chosen independently and uniformly on the region in which the nodes move. The speed is chosen from a uniform distribution in the interval $(\mathrm{v} 0, \mathrm{v} 1)$. Once the pause time expires, the node chooses a new destination, speed, and pause time. The movement of a node from the starting position (waypoint) to its next destination (waypoint) is known as one movement epoch. Moreover, the model permits nodes to pause between two sequential travels for a certain period of time. This period (Pause Time) is habitually fixed to a constant value (Tpause) [2]. In the random waypoint model, speed and pause time are the two key parameters that decide the mobility behaviour of nodes [3].

\subsection{Random Direction Mobility Model}

The Random Direction model was created to avoid the issue of density wave caused by random waypoint mobility model. A density wave is the clustering of nodes in a single piece of the simulation field. In the random direction model the node randomly and uniformly choose a direction and it moves along until the point when it achieves the boundary, when the node achieves the boundary of the simulation field and stops in the pause time, then it randomly chooses another direction to the movement. In such way, the nodes are uniformly disseminated inside the simulation field [4].

\subsection{Random walk Mobility Model}

The Random Walk Model also called Brownian motion was described mathematically by Einstein 1926 [5], to rival the unpredictable movement of particles in physics. since some mobile nodes are accepted to move in a sudden way, Random Walk Mobility Model is proposed to imitate the unpredictable movement of nodes. The Random Walk Model has similarities with The Random Waypoint. We can think the Random Walk Model as the particular Random Waypoint Model with zero pause time. in any case, each movement in the Random Walk Mobility Model happens in either a constant time interval $t$ or a constant distance travelled $d$. For each new interval $t$, every node randomly and uniformly chooses its new direction $D_{t}$ from $[0,2 \pi]$. In like way, the new speed takes from a uniform distribution or a Gaussian distribution from $\left[0, S_{\text {max }}\right]$. Subsequently, also time interval $\mathrm{t}$, the node moves with the speed (s) vector $\left(S_{t} \cos D_{t}, S_{t} \sin D_{t}\right)[6]$. The Random Walk Model is a memory less mobility process where the information about the past status isn't utilized for the future choice. in other words, the present speed is autonomous with its past speed and the future speed is also independent with its present speed [7].

\subsection{Steady-State Random Waypoint Mobility Model}

This model in view of Random Waypoint Mobility (RWM) Model for case when speed, pause and position are uniformly distributed random variables. The distinction is that the choice of this parameter is not from uniform distribution but rather from stationary distribution of RWM model.

\subsection{Stationary Distribution (with Pausing)}

Suppose $\mathrm{S}$ is the speed of a Node and $(\mathrm{X}, \mathrm{Y})$ is the $\mathrm{x}$ and $\mathrm{y}$ coordinats of the node. Let $\mathrm{f}(\mathrm{s})$ be the stationary density of S and let $g(x)$ be the stationary density of X (and of Y, since the X-coordinate and ycoordinate are identically distributed) if there is no pausing. The expressions for $f(s)$ and $g(x)$ are given in (1) and (2). 


$$
\begin{gathered}
f(s)=\left\{\begin{array}{cc}
\frac{1}{s \log \left(v_{1} / v_{0}\right)} & v_{0}<s<v_{1} \\
0 & \text { otherwise }
\end{array}\right. \\
g(x)=\int_{0}^{x} \int_{x}^{1} \int_{0}^{1} \int_{0}^{1} g\left(x \mid x_{1}, x_{2}\right) h\left(x_{1}, x_{2}, y_{1}, y_{2}\right) \\
+\int_{0}^{x} \int_{x}^{1} \int_{0}^{1} \int_{0}^{1} g\left(x \mid x_{1}, x_{2}\right) h\left(x_{1}, x_{2}, y_{1}, y_{2}\right) \\
=2 \int_{0}^{x} \int_{x}^{1} \int_{0}^{1} \int_{0}^{1} \frac{k\left[\left(x_{2}-x_{1}\right)^{2}+\left(y_{2}-y_{1}\right)^{2}\right]^{1 / 2}}{x_{2}-x_{1}} \\
d y_{1} d y_{2} d x_{1} d x_{2}
\end{gathered}
$$

Assume that at every destination, a pause time $\mathrm{P}$ is picked by a probability density function $h(p)$. In practice, $h(p)$ is typically a uniform distribution, at the same time, on a fundamental level, this need not be so. additionally, accept that $\mathrm{p}$ is independent of $\mathrm{S}, \mathrm{X}$, and $\mathrm{Y}$.

We begin by computing the proportion of time that the node is paused. We refer to the travel between two consecutive destinations as an excursion. Let $\mathrm{T}$ be the time spent traveling on an excursion, excluding pause time. By definition of the Random Waypoint mobility model, the nodes movement consists of periods of travel alternating with periods of pausing. Let $E(p)$ denote the expected length of a pause and let $E(T)$ denote the expected time elapsed in traveling between two pauses. The long-run proportion of time spent paused is.

$$
P_{\text {pause }}=\frac{E(P)}{E(P)+E(T)}
$$

The expected pause time, $E(P)$, depends on the distribution from which the pause time is sampled and is given by

$$
E(P) \int_{0}^{\infty} p h(p) d p
$$

Recall that $h(p)$ denotes the probability density function of the pause time P. Let $H(p)$ be the cumulative distribution function associated with $h(p)$. By a fundamental result in renewal theory [8], the cumulative distribution function of $P_{0}$ is

$$
H_{0}(P)=\frac{\int_{0}^{p}[1-H(t)] d t}{E(P)}
$$

To sample $P_{0}$ from the cumulative distribution function $H_{0}(p)$, it is necessary to compute the inverse $H_{0}{ }^{-1}$. Then, choose $\mathrm{U}$ uniformly on $(0,1)$ and let $P_{0}=H_{0}^{-1}(U)$. The initial position $\left(x_{1}, y_{1}\right)$, is chosen uniformly on the unit square. The node remains at $\left(x_{1}, y_{1}\right)$ for a length of time equal to $P_{0}$. The initial speed of the node, once the period $P_{0}$ is over, is chosen uniformly on $\left(v_{0}, v_{1}\right)$. As an example, suppose the pause time $\mathrm{P}$ is distributed uniformly on $\left(P_{\min }, P_{\max }\right)$. The cumulative distribution of $\mathrm{P}$ is

$$
H(t)=\left\{\begin{array}{lc}
0 & t<P_{\min } \\
\frac{p-P_{\min }}{P_{\max }-P_{\min }} & P_{\min } \leq t \leq P_{\max } \\
1 & t>P_{\max } .
\end{array}\right.
$$

And the expectation of $\mathrm{P}$ is $E(P)=\left(P_{\min }+P_{\max }\right) / 2$.Computing the integral in (5) yield

$$
H_{0}(p)=\left\{\begin{array}{lc}
\frac{2 p}{P_{\min }+P_{\max }} & 0<p<P_{\min } \\
\frac{-p^{2}+2 p P_{\max }-P^{2} \min }{P^{2} \max -P^{2} \min } & P_{\min } \leq p \leq P_{\max } \\
1 & p>P_{\max } .
\end{array}\right.
$$

Inverting yields 


$$
H_{0}^{-1}(u)= \begin{cases}\frac{u\left(P_{\min }+P_{\max }\right)}{2} & 0<u<\frac{2 P_{\min }}{P_{\min }+P_{\max }} \\ P_{\max }-\sqrt{(1-u)\left(P^{2} \max -P_{\min }^{2}\right)} \frac{2 P_{\min }}{P_{\min }+P_{\max }} \leq u \leq 1\end{cases}
$$

To verify that this is the correct inverse, one may check that $\mathrm{H}_{0}\left(\mathrm{H}_{0}{ }^{-1}(\mathrm{u})\right)=\mathrm{u}$ for $0<\mathrm{u}<1$. Therefore, to choose $\mathrm{P}_{0}$, choose $\mathrm{U}$ uniformly on $(0,1)$ and let

$$
P_{0}=H_{0}^{-1}(U)
$$

As a special case, when $P_{\text {min }}=0, P_{0}=P_{\text {max }}(1-\sqrt{1-U)}$. To compute $\mathrm{E}(\mathrm{T})$, let $\mathrm{L}$ be the length of an excursion and let $\mathrm{S}$ be the speed of the node on that excursion. Note that, according to the Random Waypoint mobility model, $\mathrm{S}$ is chosen from a uniform distribution on (v0, v1) at the beginning of each excursion. Then, $\mathrm{T}=\mathrm{L} / \mathrm{S}$ and

$$
E(T)=\mathrm{E}(\mathrm{L} / \mathrm{S})=E(L) E(L / S)
$$

Since $\mathrm{L}$ and $\mathrm{S}$ are independent. We first compute $E(1 / S)$ :

$$
E(1 / S)=\int_{v_{0}}^{v_{1}}(1 / s) \frac{1}{v_{1}-v_{0}} d s=\frac{\log \left(v_{1} / v_{0}\right)}{v_{1}-v_{0}}
$$

To compute $\mathrm{E}(\mathrm{L})$, note that $\mathrm{L}$ is the distance between two points chosen independently and uniformly. Therefore,

$$
\begin{gathered}
E(L)=\int_{0}^{1} \int_{0}^{1} \int_{0}^{1} \int_{0}^{1}\left[\left(x_{2}-x_{1}\right)^{2}+\left(y_{2}-y_{1}\right)^{2}\right]^{\frac{1}{2}} \\
d x_{1} d x_{2} d y_{1} d y_{2}
\end{gathered}
$$

We compute this integral numerically to obtain

$$
E(L)=0.521405
$$

Therefore,

$$
E(T)=0.521405 \frac{\log \left(v_{1} / v_{0}\right)}{v_{1}-v_{0}}
$$

The result in (14) has also been shown in [9]. Note for a square of side a, $E(L)=0.521405 a$, a result in (7) can be used to show that

$$
\mathrm{E}(\mathrm{L})=\frac{1}{6}\left[\frac{b^{2}}{a} \log \left(\sqrt{\frac{a^{2}}{b^{2}}+1}+\frac{a}{b}\right)+\frac{a^{2}}{b} \log \left(\sqrt{\frac{b^{2}}{a^{2}}+1} \frac{b}{a}\right)\right]+\frac{1}{15}\left(\frac{a^{3}}{b^{2}}+\frac{b^{3}}{a^{2}}\right)-\frac{1}{15} \sqrt{a^{2}+b^{2}}\left(\frac{a^{2}}{b^{2}}+\frac{b^{2}}{a^{2}}-3\right)
$$

Now, let $f_{1}(\mathrm{~s})$ be the density of $\mathrm{S}$. The conditional density $f_{1}$ (s|Paused) is equal to the density $f_{1}(\mathrm{~s})$ in (1). Furthermore, if the node is paused, the speed is zero with probability 1 . Therefore,

$$
\begin{aligned}
& \mathrm{f}_{1}(\mathrm{~s})=\mathrm{f}_{1}(\mathrm{~s} \mid \text { Paused }) P_{\text {pause }}+\mathrm{f}_{1}(\mathrm{~s} \mid \text { NotPaused })\left(1-\mathrm{P}_{\text {pause }}\right) \\
& =\left\{\begin{array}{lc}
0 & s<0 \\
P_{\text {pause }} & \mathrm{s}=0 \\
0 & 0 \leq \mathrm{s} \leq v_{0} \\
\frac{1-P_{\text {pause }}}{\mathrm{s} \log \left(v_{1} / v_{0}\right)} & v_{0} \leq \mathrm{s} \leq v_{1} \\
0 & s>v_{1}
\end{array}\right.
\end{aligned}
$$

Let $g_{1}(\mathrm{x})$ be the density of $\mathrm{X}$ (and of $\mathrm{Y}$ since the $\mathrm{x}$-coordinate and $\mathrm{y}$-coordinate are identically distributed). Then,

$$
g_{1}(\mathrm{x})=g_{1}(\mathrm{x} \mid \text { Paused }) P_{\text {pause }}+g_{1}(\mathrm{x} \mid \text { NotPaused })\left(1-P_{\text {pause }}\right)
$$


where $g_{1}$ (x|Not Paused) is equal to the density $g(x)$ in $(2)$, and $g_{1}$ (x|Paused) is the uniform density on $(0,1)$ since the coordinates of path endpoints are uniformly distribution [10].

\section{OPTIMIZED LINK STATE ROUTING PROTOCOL}

The Optimized Link State routing (OLSR) is a table-driven pro-active protocol. It uses the link-state scheme in an optimized manner to diffuse topology information. In a classic link-state algorithm, because of its proactive (N. Meghanathan,2010) nature the routes are always immediately available when needed. It utilizes periodic exchange of messages to keep up topology information of the network at each node. Besides, OLSR utilizes Multipoint Relays (MPR) to reduce the possible overhead in the network protocol in Figure 1. It limits flooding of this control traffic by utilizing just the chose nodes, called multipoint relays, to diffuse its messages in the network. Just the multipoint relays of a node retransmit its broadcast messages [11].

\subsection{Multipoint Relays}

The idea of multipoint relays (MPR) is to reduce the information exchange overhead. Each node in the network chooses a set of nodes in its neighborhood, which retransmit its packets. This set of chose nodes is known as the multipoint relays (MPRS) of that node. The neighbors of any node $\mathrm{N}$ which are not in its MPR set, read and process the packet but don't retransmit the broadcast packet received from node N. For this reason, every node maintains a set of its neighbors which called MPR selectors of node. This is accomplished by choosing neighbors as multipoint relays (MPRS). Each node calculates its own particular set of MPRS as a subset of its symmetric neighbor nodes picked with the goal that all two hop neighbors can be reached by MPR. This implies for each node $\mathrm{N}$ in the network [12]. Figure 1 shows flooding a packet in a wireless multihop network from the center node using MPRs (blue)

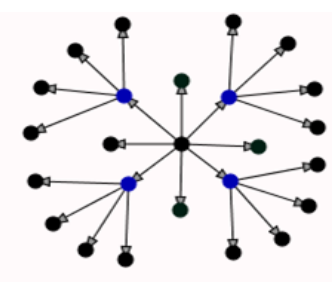

- MPR (Retransmission node)

Figure 1. Flooding a packet in a wireless multihop network from the center node using MPRs (blue)

Node A selects the blue nodes as MPRs in Figure 2. Along these lines every one of the two hop nodes can be reached by MPR. Node B won't retransmit traffic from A that will be flooded, each node must distinguish the neighbor nodes with which it has a direct and bi-directional link., all links must be checked in the two directions.

To achieve this, every node periodically broadcasts its HELLO messages, containing the information about its neighbors and their link status. These control messages are transmitted in the broadcast mode. These are gotten by all one-hop neighbors. A HELLO message contains [13]:

The list of addresses of the neighbors to which there exists a valid bi-directional link. The list of addresses of the neighbors which are heard by this node (a hello has been received) yet the link isn't yet approved as bi-directional, if a node discovers his own particular address in a HELLO message, it considers the link to the sender node as bi-directional.

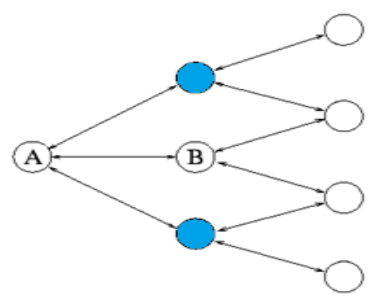

Figure 2. Node A has selected the blue nodes as its MPRs 
This HELLO messages allow every node to know its tow hops neighbors. These chose multipoint relays are demonstrated in the HELLO messages with the link status MPR. On the gathering of HELLO messages, every node can develop its MPR selector table with the nodes who have chosen it as a multipoint relay [14] Performance Metrics So as to conduct this study of OLSR, its performance has been analyzed under four mobility models (Random Waypoint, Random Direction, Random Walk, and Steady-State Random Waypoint) with respect to tree performance metrics based on CBR traffic. Simulations have been carried out by Network Simulator 3 (NS-3).

\section{RESULTS AND ANALYSIS}

In this section, we show the results achieved using OLSR routing protocol with the mobility models that we have already introduced in above sections. This simulation is done on a mobile ad hoc network with varying the number of nodes. In the first place we have to introduce the simulation metrics and simulation tools of simulation then we have to analyse the results.

\subsection{Simulation Metrics and Tools \\ 4.1.1 Packet Delivery Ratio}

Packet Delivery Ratio is a very important factor to measure the performance of routing protocol. It is defined as the ratio of number of packets received by the destination to the number of packets sent from the source. The performance is better when the packet delivery ratio is high. Mathematically it can be shown as the equation below [15-16].

$$
P D R=\frac{\sum \text { packets received by destination nodes }}{\sum \text { packets sent by source nodes }}
$$

\subsubsection{Throughput}

Throughput is defined as amount of data per unit time that has been delivered to one node from another. It is calculated in Kbps. Throughput include frequent topology changes, unreliable communication, limited bandwidth and limited energy. A high throughput network is desirable. It can be shown as equation below [16-17].

$$
\begin{aligned}
\text { Throughput }= & (\text { Packet received size } / \text { Simulation stop time }- \text { Simulation start time }) \\
& *(8 / 1000)
\end{aligned}
$$

\subsubsection{End-to-End delay}

End-to-End delay is the average time between transmission of a packet and its reception, the time it takes the packet to achieve the destination after it leaves the source. The deadline for the end-to-end delay includes the routing and other various delays, such as the transmission delay, propagation delay and delay queue. The deadline for the end-to-end is calculated as follows [17]:

$$
\text { End to End delay }=\frac{\sum \text { delay }}{\sum \text { packets received }}
$$

\subsubsection{Network Simulator 3}

Network Simulator 3 is a discrete-event network simulator, directed principally for examine and instructive utilize. NS-3 is free programming, authorized under the GNU GPLv2 license. The objective of the NS3 project is to build up a favoured, open simulation environment for networking research, it ought to be lined up with the simulation needs of current networking research and ought to empower group commitment, peer survey, and approval of the product. The NS-3 simulation center backings investigate on both IP and non-IP based networks [18-21].

\subsubsection{ECLIPSE}

ECLIPSE is a multi-language software programming environment containing an incorporated advancement condition (IDE) and an extensible programming. Begun by IBM (Canada) in late 1990s. It is composed essentially in Java and can be utilized as a part of different languages also, including $\mathrm{C}, \mathrm{C}++$, COBOL, Python, Perl, PHP, and others. We utilized module Eclipse CDT for $\mathrm{C} / \mathrm{C}++$ codes, since ns-3 executed in $\mathrm{C}++[22-25]$. 


\subsection{Simulation Results}

\subsubsection{Scenario 1: Varying the Number of Nodes}

In the scenario, we simulated nodes traveling in a $500 \mathrm{~m} \times 1500 \mathrm{~m}$ region see Table 1 . Our simulation was of the fourth mobility models the first one is the Random Waypoint mobility model, followed by SteadyState Random Waypoint, Random Walk, and Random Direction, all destinations were chosen uniformly on the region. The nodes travelled for 1000 seconds [26]. The location of the node was updated once per second. The comparison graphs of different mobility models are obtained in Figures 3, 4 and 5. It shows the impact of mobility models on OLSR based [27]on three performance metrics: Packet Delivery Ratio (PDR), Throughput and End-to-End delay. with varying number of nodes connected [28-31].

Table 1. Simulation environment

\begin{tabular}{cc}
\hline Parameter & Value \\
\hline Channel type & Wireless \\
Routing protocol & OLSR \\
Traffic type & CBR \\
Time of simulation & $1000 \mathrm{~s}$ \\
X and Y dimensions of topography & $500 \mathrm{~m} \times 1500 \mathrm{~m}$ \\
MAC protocol & $802.11 \mathrm{Ext}$ \\
Number of nodes & $20,4,60,80,100$ \\
Speed & $20 \mathrm{~m} / \mathrm{s}$ \\
\hline
\end{tabular}

\section{A. Packet Delivery Ratio (PDR)}

Figure 3 present the performance of mobility models i.e. Random Waypoint, Steady-State Random Waypoint, Random Walk, Random Direction, with varying the number of nodes in terms of Packet delivery ratio, under OLSR routing protocol. The graph shows that Random Waypoint has better PDR values followed by Steady-State Random Waypoint and Random Walk then random direction which has the lowest values. Also, the graph clearly shows that when the number of nodes increment, the PDR of the four mobility models raise.

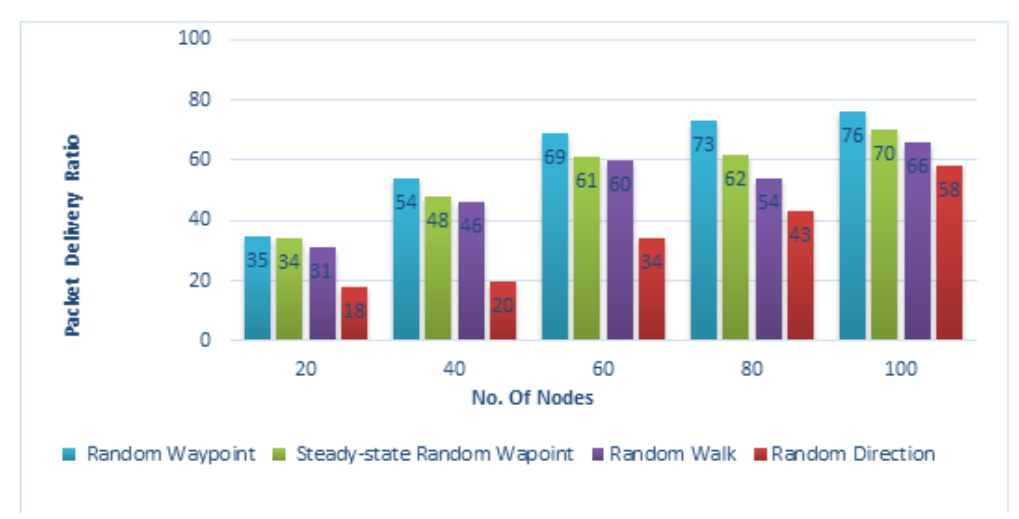

Figure 3. PDR of OLSR using four mobility models over number of nodes in MANET

\section{B. Throughput}

As we see in Figure 4 the throughput of OLSR using four mobility models decreases slowly by increasing nodes density. The throughput increments until the 80 first nodes and rest stable between the 80 and 100 nodes. Generally, the optimal throughput is got with Random waypoint mobility model followed by the Random walk and Steady-State Random Waypoint better than the previous in some cases after that the random direction, the Random Direction exhibits low values of throughput. we can conclude that the optimal throughput is got when associating the OLSR with Random Waypoint mobility model. OLSR performed better in term of throughput when small density of nodes used. 


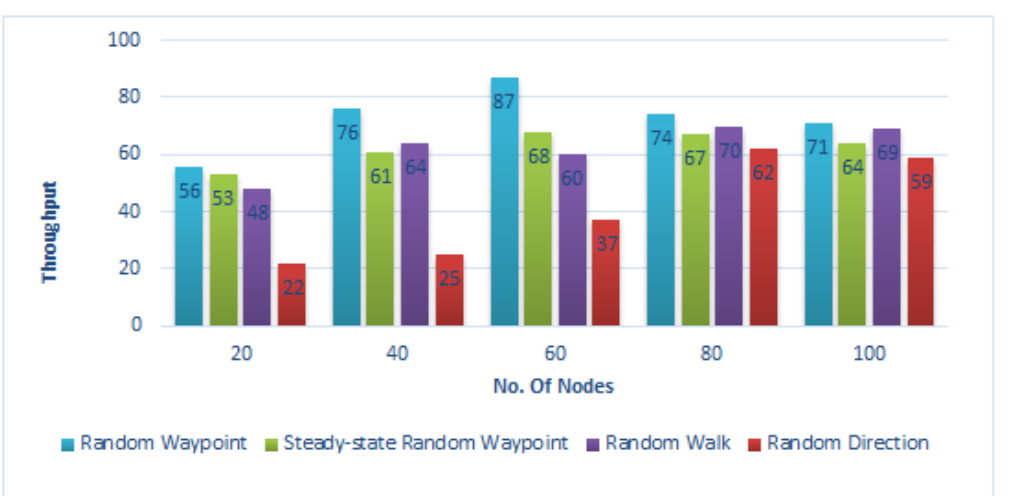

Figure 4. Throughput of OLSR using four mobility models over number of nodes in MANET

\section{End-to-End delay}

Figure 5 shows the effect of number of nodes on the End-to-End Delay. As shown in the graph, the optimal delay is achieved with OLSR under Steady- State Random waypoint is less than that got with Random Waypoint mobility model and highest when Random Direction mobility model is used. Random walk is better than Random Direction with respect to End-to-End Delay. under the OLSR routing protocols, Steady-State Random Waypoint outperforms Random Waypoint in terms of End-to-End Delay in Figure 5. Because the delay achieved by OLSR associated with Steady-State Random Waypoint is less than that got with Random Waypoint and the other mobility models, it is suggested to use this protocol in the real time applications sensitive to the delay.

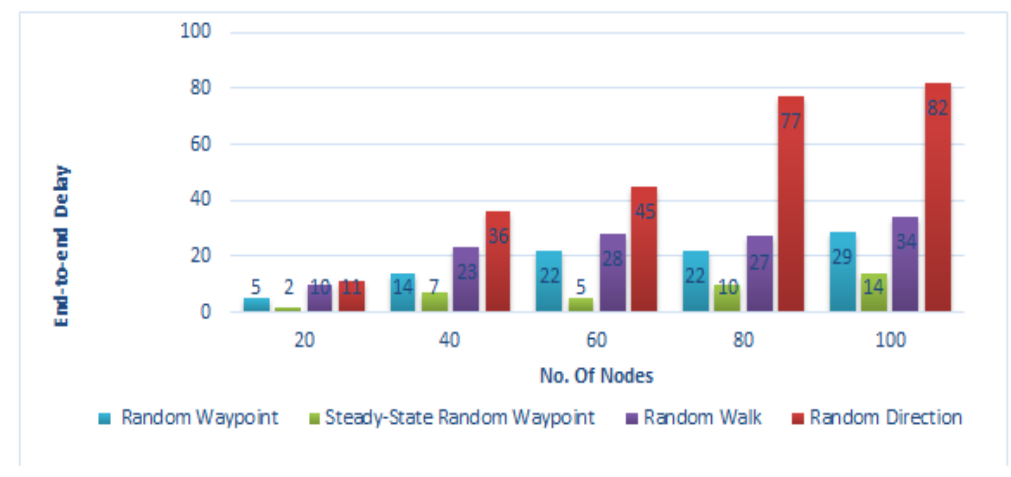

Figure 5. End-to-End Delay of OLSR using four mobility models over number of nodes in MANET

\subsubsection{Scenario 2: Varying the Speed of Nodes}

The routing protocol simulated was the Optimized Link State Routing Protocol (OLSR) which determines routes on demand, Figures 6, 7 and 8 present the impact of mobility models on OLSR based on three performance metrics: Packet Delivery Ratio (PDR), Throughput and End-to-End delay. with varying the speed of nodes connected. Table 2 shows simulation environment.

Table 2. Simulation Environment

\begin{tabular}{cc}
\hline Parameter & Value \\
\hline Channel type & Wireless \\
Routing protocol & OLSR \\
Traffic type & CBR \\
Time of simulation & $1000 \mathrm{~s}$ \\
Queue length & 2048 Bytes \\
X and Y dimensions of topography & $500 \mathrm{~m} \times 1500 \mathrm{~m}$ \\
MAC protocol & $802.11 \mathrm{Ext}$ \\
Number of nodes & 60 \\
Speed & $20,40,60,80,100 \mathrm{~m} / \mathrm{s}$ \\
\hline
\end{tabular}

Link-state QoS routing protocol under various mobility models (Safaa Laqtib) 


\section{A. Packet Delivery Ratio (PDR)}

Figure 6 depicts the Packet Delivery ratio of OLSR protocol under the four mobility models. As is evident from the figure, Steady-State Random Waypoint mobility model provides better Packet Delivery ratio than the other three mobility models, Random Waypoint provides better packet delivery ratio than Random Walk. The performance for the Steady-State Random Waypoint model and Random Waypoint is stable between $60 \mathrm{~m} / \mathrm{s}$ and $80 \mathrm{~m} / \mathrm{s}$, whereas Random Direction exhibits inconsistent packet delivery ratio with varying speed.

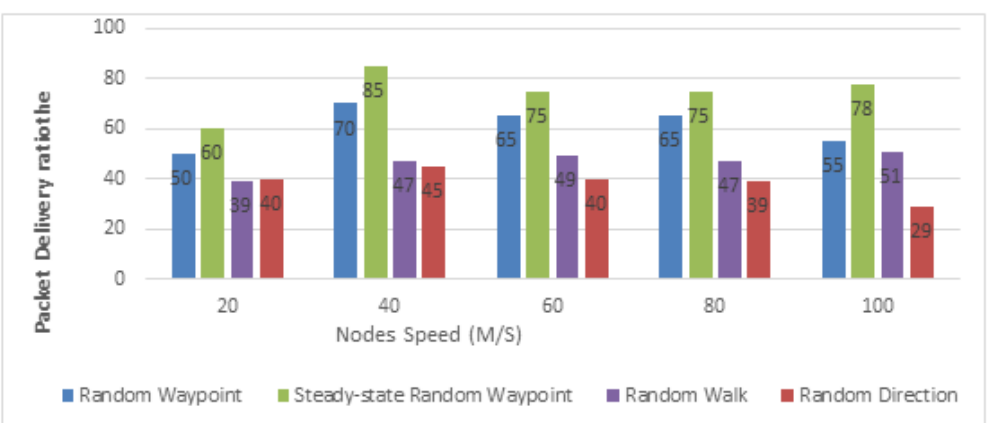

Figure 6. PDR of OLSR using four mobility models over speed of nodes In MANET

\section{B. Throughput}

The simulation results obtained using OLSR with Random Waypoint, Steady-State Random Waypoint, Random Walk and Random Direction mobility models over varying node speed are shown in Figure 7. Figure. 7 presents the results of Throughput for varying node speed from $20 \mathrm{~m} / \mathrm{s}$ to $100 \mathrm{~m} / \mathrm{s}$. It is evident that the performance of OLSR with respect to Throughput using Steady-State Random Waypoint increases as the node speed increase, Random Waypoint and Random Walk mobility models is almost similar with very little difference. But as the node speed increases the Throughput using Random Walk mobility model is found to be consistent whereas Random Waypoint shows decline in the Throughput when the speed reaches $80 \mathrm{~m} / \mathrm{s}$. In the case of Random direction mobility model, as the node speed increases there is substantial drop in the Throughput. we can conclude that the optimal Throughput is got when associating the OLSR with the Steady-State Random Waypoint mobility model.

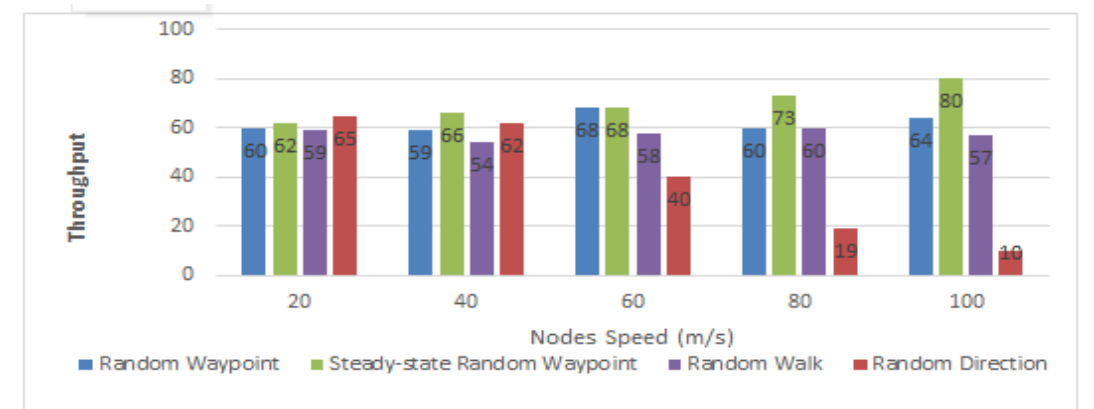

Figure 7. throughput of OLSR using four mobility models over speed of nodes in MANET

\section{End-to-End delay}

The delay incurred by OLSR protocol using the four mobility models is shown in Figure 8 . With reference to End-to-End delay, the OLSR protocol using Steady-State Random Waypoint mobility model exhibits least delay and it is consistent with increase in speed. In the case of Random Waypoint mobility model, delay is less than Random Walk model, but it is far better than Random Direction mobility model, which exhibits high End-to-End delay as the node speed increases. Steady-State Random Waypoint mobility performed better in term of End-to-End delay. 


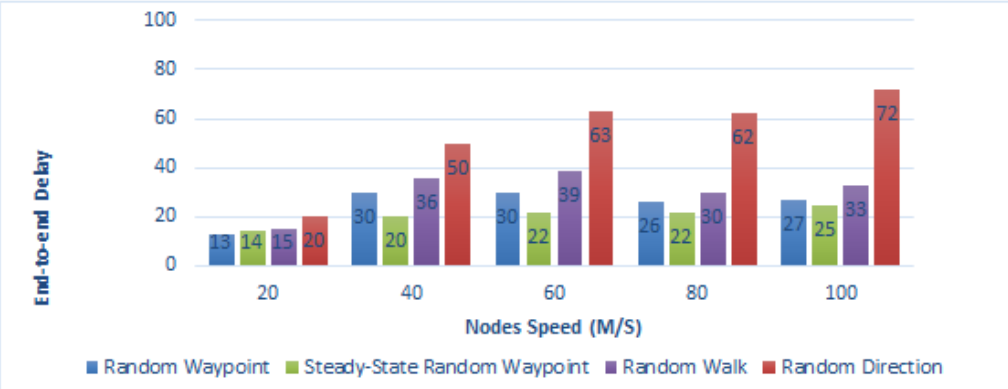

Figure 8. End-to-End Delay of OLSR using four mobility models over speed of nodes in MANET

\section{CONCLUSION}

This paper presents the impact of different mobility models (Random Waypoint, Steady-State Random Waypoint, Random Walk, and Random Direction) on OLSR routing protocol, in mobile ad hoc network with varying respectively the number of nodes and the speed of nodes. The performance was measured under three QoS (Quality of Service) metrics such as the Packet Delivery Ratio (PDR), Throughput, End-to-End delay. From the simulation results, the performance of Random Direction mobility model does not seem to be better than the other three mobility models. It provides a low Packet Delivery Ratio, low Throughput and higher End-to-End delay. As far as Random Walk and Random Waypoint is considered, OLSR with Random Waypoint provides good PDR and Throughput and Steady-State Random Waypoint provides low End-to-End delay followed by Random Waypoint and Random Walk. It is evident from the results that Random Waypoint is the perform mobility model for OLSR in MANET when using Packet Delivery Ratio and Throughput with varying the number of nodes and Steady-State Random Waypoint presents better result when using End-to-End delay with varying the number speed of nodes. We can conclude that there is significant impact of the mobility model on the routing protocol. However, in future we will try to study the impact of Steady-State Random Waypoint on other protocols.

\section{REFERENCES}

[1] A. Jain, B. Buksh., "Solutions for Secure Routing in Mobile Ad Hoc Network (MANET): A Survey,"Imperial Journal of Interdisciplinary Research. no. 4, pp. 5-8, 2016.

[2] M Nisar,A. Mehmood, A. Nadeem A. Ahsan Sarim M., “ A Two-Dimensional Performance Analysis of Mobility Models for MANETs and VANETs," Research Journal of Recent Sciences, Vol. 3, No. 5, pp. 94-103, 2014.

[3] K. Devarajan, V.Padmathilagam., "Performance Evaluation of MANET Routing Protocol Using Random Way Point and Manhattan Mobility Models," Middle-East Journal of Scientific Research, 24 (8): 2523-2530, 2016

[4] N. Meghanathan., " Impact of the Gauss-Markov mobility model on network connectivity, lifetime and hop count of routes for mobile Ad hoc networks," J. Networks, Vol. 5, No. 5, pp. 509-516, 2010.

[5] Birendranath Ghosh, " Random Distances within a Rectangle and between Rectangles Bull," Calcutta Math. Soc; Vol. 2, pp. 17-24, 1951.

[6] P Katkar, S., " Comparative Study of Network Simulator: NS2 and NS3," Int. J. Adv. Res. Comput. Sci. Softw. Eng,Vol. 6, No. 3, pp. 608-612, 2016.

[7] S Gowrishankar, T G. Basavaraju, Subir Kumar Sarkar., "Effect of Random Mobility Models Pattern in Mobile Ad hoc Networks," IJCSNS International Journal of Computer Science and Network Security, VOL.7 No.6, 2017.

[8] Chenna Reddy, P Chandrasekhar Reddy., "Mobile Ad-hoc network: Working of routing protocols and applications,"Proceedings. IEEE COMMUNICATIONS LETTERS, Vol. 17, No. 6, pp. 1311-4360 2016.

[9] C. Bettstetter, Hartenstein, H. Perez-Costa., " Stochastic properties of the random waypoint mobility model epoch length, direction distribution, and cell change rate”. Proceedings MSWiM '02. 5th ACM Int. Work. Model. Anal. Simul. Wirel. Mob. Syst. pp. 7-14, 2002.

[10] W. Navidi, and T. Camp, sudhir KUMAR sharma.Relative., " Stationary Distributions for the Random Waypoint Mobility Model.," Proceedings IEEE Trans. Mob. Comput, Vol. 3, No. 1, pp. 99-108.

[11] pankaj kumar varshney, agrawal, g.s, and sudhir KUMAR sharma.Relative., " Performance Analysis of Proactive Routing Protocols wireless Ad hoc Networks using Varying Node Density. Invertis Journal of Science and Technology, Vol. 9, No. 3, 2016.

[12] Mohammed Ahmed Jubair, Shihab Hamad Khaleefah, Avon Budiyono, Salama A. Mostafa., “ Performance Evaluation of AODV and OLSR Routing Protocols in MANET Environment," International Journal On Advanced Science Engineering And Information Technology, vol8 no 42018.

[13] Shihab A. Hameed, Mohamed Hadi Habaebi, Alaa Haddad., "Application of Mobile Cloud Computing in Emergency Health Care," Bulletin of Electrical Engineering and Informatics (BEEI), Vol 8, No 3: September 2019. 
[14] Ngan Nguyen, Quoc Cuong Nguyen, Minh Thuy Le., “ A Novel Autonomous Wireless Sensor Node for IoT Applications," TELKOMNIKA (Telecommunication, Computing, Electronics and Control) International 2019.

[15] Mansour Bechar, Abdeldjebar Hazzab, Mohamed Habbab, Pierre Sicard., "Reduced-Order Observer For Real-Time Implementation Speed Sensorless Control of Induction Using RT-LAB Software," International Journal of Power Electronics and Drive Systems, Vol 10, No 3: September 2019

[16] M.Bheemalingaiah, M. M Naidu, Sreenivasa Rao, D. P.Sreenivasa Moorthy., "Survey of Routing Protocols, Simulation Tools and Mobility," International Journal of Innovations \& Advancement in Computer Science IJIACS, Vol 6, Issue 11 November 2017.

[17] Hasitha Priyadarshani, Nipuna Jayasekara, Lahiru Chathuranga, Krishnadeva Kesavan, Chamira Nawarathn, KalpaKalhara Sampath,Cethana Liyanapathirana, Lakmak Rupasinghe., "Enhancing the Security of OLSR Protocol Using Reinforcement Learning," Proceedings. IEEE National Information Technology Conference (NITC), Colombo, Sri Lanka, 13-15 September, 2017.

[18] Saima Zafar, Hina Tariq, Kanza Manzoor., "Throughput and Delay Analysis of AODV, DSDV and DSR Routing Protocols in Mobile Ad Hoc Networks," International Journal of Computer Networks and u Applications (IJCNA), Vol 3, Issue 2, March-April 2016.

[19] Atul Sharma, Piyush Gupta, Jitender Grover. , "IOSR: Configuration of ONE Simulator using Eclipse," Journal of Electronics and Communication Engineering (IOSR-JECE), 2016.

[20] Manhal Alhilali, Mustafa Ghanim, Jafri Din, Hong Yin Lam., "A Methodology for Precise Estimation of Rain Attenuation on Terrestrial Millimetre Wave Links from Raindrop Size Distribution Measurements," TELKOMNIKA (Telecommunication, Computing, Electronics and Control), Vol 17, No 5 October 2019.

[21] Amerrul Zabri, M. K. A. Rahim, F. Zubir, N. M. Nadzir, H. A., " Majid Fractal Yagi-Uda Antenna for WLAN Applications," TELKOMNIKA (Telecommunication, Computing, Electronics and Control) International, Vol 17, No 5 October 2019.

[22] Sridhar Babu Gurijala, K S Srikanth, Ramchandra Nittala, G. Rohit Reddy.,"Transmission System Regularization with 5-Level Cascaded IPFC, "Vol 10,No3,september 2019.

[23] M - yahya., "Performance Analyses On Fluidized Bed Dryer Integrated Biomass Furnace With and Without Air Preheater For Paddy Drying, " International Journal of Power Electronics and Drive Systems (IJPEDS), Vol 10,No3,september 2019.

[24] G Kanimozhi., "Pre-scheduled load shutdown in Microgrid utilizing computerized surveillance system," Indonesian Journal of Electrical Engineering and Computer Science (IJEECS), Vol 15, No 3, October 2019.

[25] Idrissa Abubakar, Jafri Bin Din, Lam Hong Yin, Manhal Alhilali., " Rain attenuation in broadband satellite service and worst month analysis", Indonesian Journal of Electrical Engineering and Computer Science (IJEECS), Vol 15, No 3 September 2019.

[26] Muhammad Afiq Abdul Aziz, Norhudah Seman, Tien Han Chua., "Microstrip antenna design with partial ground at frequencies above $20 \mathrm{GHz}$ for $5 \mathrm{G}$ telecommunication systems ", Indonesian Journal of Electrical Engineering and Computer Science (IJEECS), Vol 15, No 3 September 2019.

[27] Adib Othman, Nur Ishmah Sabirah Shaari, Abdullah M. Zobilah, Noor Azwan Shairi, Zahriladha Zakaria., "Design of Compact Ultra Wideband Antenna for Microwave Medical Imaging Application ”, Indonesian Journal of Electrical Engineering and Computer Science (IJEECS), Vol 15, No 3 September 2019.

[28] Nurlan Batayev, "Axial Compressor Fouling Detection for Gas Turbine Driven Gas Compression Unit", Indonesian Journal of Electrical Engineering and Computer Science (IJEECS), Vol 15, No 3 September 2019.

[29] djelloul kheira., "Performance of channel selection used for Multi-class EEG signal classification of motor imagery", Indonesian Journal of Electrical Engineering and Computer Science (IJEECS), Vol 15, No 3 September 2019.

[30] Sk Hasane Ahammad, "Automatic Segmentation of Spinal Cord Diffusion MR Images for disease location finding", Indonesian Journal of Electrical Engineering and Computer Science (IJEECS), Vol 15, No 3 September 2019.

[31] Abil Fathir Majid, Yulisdin Mukhlis “Aperture Coupling Rectangular Slotted Circular Ring Microstrip Patch ”, Indonesian Journal of Electrical Engineering and Computer Science (IJEECS), Vol 15, No 3 September 2019 Antenna. 